\title{
Review of: "Thawing Yedoma permafrost is a neglected nitrous oxide source"
}

\author{
Paul Schuster ${ }^{1}$ \\ 1 United States Geological Survey
}

Potential competing interests: The author(s) declared that no potential competing interests exist.

Post-publication comments; Schuster 1/21/22:

Thawing Yedoma permafrost is a neglected nitrous oxide source, M. E. Marushchak,et. al. NATURE COMMUNICATIONS | (2021) 12:7107 | https://doi.org/10.1038/s41467-021-27386-2 | www.nature.com/naturecommunications

Summary:

Although there is no evidence of N2O emissions increasing globally since 1990 (EPA), this study has identified drying and revegetation with grasses and structural changes of the microbial community responsible for $\mathrm{N}$ cycling as mechanisms for increased $\mathrm{N} 2 \mathrm{O}$ emissions from thawing Yedoma at two locations in Siberia. Logistics and economics often prohibit wide-scale studies of this nature. As with many Arctic studies there is always the question of scaling up. A basic assumption is usually made that the quantification and qualification of identified processes transfer to other environments with similar characteristics. Despite these unavoidable shortfalls, these results advance the state of the science and understanding of the importance of N2O emissions as a positive feedback to global warming highlighting the importance of extra $\mathrm{N}$ availability from thawing Yedoma permafrost.

Additional comments:

Why did the authors single out N2O6 when all nitrous oxides are considered GHG's? Is it simply because N2O6 is 300X more powerful as a GHG than CO2? The reader is left hanging here. How much N2O transforms to N2O6? How stable is $\mathrm{N} 2 \mathrm{O} 6$ and how long does it cycle in the atmosphere?

In Results and discussion, the following wording

"At the Kurungnakh exposure, the N2O fluxes from thawed Yedoma surfaces were highly variable (63 (-196286) $\mu \mathrm{gNm}-2$ day -1 ; median with (range)), at the high-end exceeding the typical fluxes from permafrost-affected soils (38 (6-189) $\mu \mathrm{gNm}-2$ day-1; median with (25th-75th percentiles); ref. 6 ) by two orders of magnitude" 
Makes it sound very alarming by using "two orders of magnitude" when more realistically and statistically, it is only a 2-fold increase on average. It seems to me the authors are "cherry-picking" their verbiage to sound what might be considered over-stated alarms.

$\mathrm{N} 2 \mathrm{O}$ vs $[\mathrm{C}]$ and $\mathrm{C}$-flux shows a significant correlation $\mathrm{R} 2=0.5, p=0.05$ and $0.4, p=0.002$, respectively. Suggesting plant derived organic C may stimulate $\mathrm{N}$ cycling processes. This is a very important observation regarding globally thawing permafrost and it should have received more discussion and explanation.

Thawing and subsequent drying of oxic permafrost (Yedema) due to a warming Earth may result in a 6-fold increase in N2O production as a positive feedback. Now that is alarming!

This is a very insightful article. 